\title{
Roll vection analysis of suggestion-induced visual field narrowing
}

\author{
H. W. LEIBOWITZ, R. B. POST, C. SHUPER'T RODEMER, \\ W. L. WADLINGTON, and R. M. LUNDY \\ Pennsylvania State University, University Park, Pennsylvania 16802
}

\begin{abstract}
Instructions to simulate visual field narrowing resulted in apparently narrow visual fields when these were evaluated by means of conventional perimetry. However, when a pattern of stripes moving around the visual axis was viewed, the magnitude of the induced change in the subjective horizontal or vertical (roll vection) was unaffected. These results demonstrate that conventional perimetric techniques may in some instances be inadequate to demonstrate functional peripheral vision. Evaluation of the peripheral visual field in perimetry, the role of peripheral vision in visually guided behavior, and the effect of stressors on peripheral visual functions are discussed.
\end{abstract}

Narrowing of the visual field is a frequently reported phenomenon in both the clinical and experimental literatures. ${ }^{1}$ Charcot (1890) made early reference to this effect (rétrécissement du champ visuel), maintaining that narrowing of the visual field was characteristic of hysteria and could serve as a reliable diagnostic sign. A similar effect is also reported in the experimental psychology and human engineering literatures, in which a number of stressors, including heat, cold, high perceptual-motor load, fatigue, and hypoxia, have been reported to produce peripheral narrowing (Leibowitz, 1973). Apparently, certain emotional states or experimentally induced stress may interfere with the reporting of peripherally presented stimuli.

It is important to note that the observer's task in traditional perimetry (Aulhorn \& Harms, 1972), that is, the detection of small static targets, may be unrelated to one of the major functions served by peripheral vision, that of orientation in space. It has been shown that the loss of fine detail resulting from image degradation does not influence orientation responses (Leibowitz, Rodemer, \& Dichgans, 1979; Post \& Leibowitz, in press; Post, Rodemer, Dichgans, \& Leibowitz, Note 1). Additionally, peripheral stimuli of which one is unaware may be used for spatial orientation during such activities as simultaneous walking and reading. Thus, the functional significance of the peripheral fields may not actually be accu-

Supported by Grant MH08061 from the National Institute of Mental Health and Grant EY03276 from the National Eye Institute. The authors wish to express their appreciation to Drs. E. R. Hilgard, R. J. Miller, and B. U. Crassini for helpful suggestions in the preparation of the manuscript. Please address requests for reprints to H. Leibowitz, Moore Building, University Park, Pennsylvania 16802 . rately reflected by verbal reports of awareness of peripheral stimuli.

Since conventional perimetry is based on subjects' verbal reports, it is highly dependent on the subjects' willingness to respond to the presence or absence of stimuli (i.e., response bias), as well as on their actual ability to detect the stimuli. One instance of apparent narrowing that presumably reflects this limitation of perimetry is that observed during hypnosis. Although subjects consistently report that they cannot see peripheral stimuli following narrowing instructions (Miller \& Leibowitz, 1976), there are problems with inferring that a real sensory loss occurs. Subjects who are not hypnotized but are only simulating demonstrate apparent visual narrowing as well indicating that the narrowing observed among hypnotized subjects may be due to demand characteristics. Such an interpretation is supported by the results of a subsequent study (Leibowitz, Lundy, \& Guez, 1980), which demonstrated that hypnotically induced visual field narrowing may vary in angular size with changes in viewing distance. A true sensory loss would exhibit a constant visual angle over different viewing distances. Thus, true sensory loss and hypnotically induced sensory loss appear identical when evaluated with conventional perimetry but may be shown to be different when evaluated by means of some less obvious procedure, such as size-distance relationships.

This limitation of classical perimetry as an assessment technique for peripheral vision may help account for the observations of Janet (1907), who found very narrow fields among hysterical patients, some as small as $5 \mathrm{deg}$, but also noted that these same patients were able to "play very cleverly at ball" in the courtyard of the Salpettrière hospital. Janet compared the hysterical patients with those who had lost peripheral vision due to retinal damage asso- 
ciated with glaucoma. In contrast to the apparently normal mobility of the hysterical patients, glaucoma patients were severely handicapped. They locomoted very slowly and made numerous eye and head movements in order to compensate for the loss of peripheral information. From these observations, Janet concluded that hysterical patients retain their mobility as a result of a "residual unconscious visual function." Within the present context, a more parsimonious explanation would be that classical perimetric techniques are insufficient to evaluate peripheral vision in such individuals.

In the present study, roll vection (the feeling of selfmotion and change in apparent vertical or horizontal produced by a stimulus rotating about the line of sight) was used to evaluate peripheral vision in subjects with hypnotically "narrowed" vision. Possible advantages of this procedure are that: (1) roll vection assesses a major function of peripheral vision, spatial orientation, which is ignored in conventional perimetry, and (2) the apparent change in vertical or horizontal produced during roll vection is quite compelling and is relatively unknown (as a perceptual phenomenon). It should therefore provide a good paradigm for peripheral visual assessment with minimal interference by response bias, demand characteristics, or subjects' expectations. In the experiment to be reported, apparent visual field narrowing was investigated with three groups of subjects-hypnotized, simulating, and instructed faking. The simulating and faking groups were included to assess the effects of demand characteristics on the verbal reports of subjects. The objective was to determine whether roll vection measures would be more resistant than traditional perimetry responses to the attempts of subjects to demonstrate visual field narrowing.

\section{METHOD}

Visual fields were determined with a Gambs perimeter with a radius of $33 \mathrm{~cm}$ and a background luminance of $.27 \mathrm{~cd} / \mathrm{m}^{2}$. The increment target consisted of a red disk subtending $1.65 \mathrm{deg}$, $1.1 \mathrm{~cd} / \mathrm{m}^{2}$, presented for $.2 \mathrm{sec}$.

The magnitude of roll vection was determined with a hemisphere, $61 \mathrm{~cm}$ in diameter. A stationary white disk, $21.8 \mathrm{~cm}$ in diameter and subtending $67 \mathrm{deg}$ of arc, was mounted in the subject's frontoparallel plane in the center of the hemisphere's inner surface. Peripherally to this disk, 16 black stripes, $1.85 \mathrm{~cm}$ in width, were mounted in a regular pattern radiating from the center of the hemisphere. When the stripes were rotated around the subject's line of sight at $60 \mathrm{deg} / \mathrm{sec}$, the stripes induced a sensation of selfmotion or roll vection in the direction opposite to the rotation. Simultaneously, a stationary bar in the center of the visual field would appear to tilt in the direction opposite to the rotation of the stripes. The magnitude of the induced roll vection was determined with the aid of a central bar, $4 \times 85 \mathrm{~mm}$, that was mounted on a white 88-mm-diam disk located $.64 \mathrm{~cm}$ in front of the stationary disk, concentric with and rotatable independently of the hemisphere. The subject's dominant eye was aligned with the axis of rotation at a distance of approximately $18 \mathrm{~cm}$ from the adjustable bar. The subject was instructed to adjust this bar manually by means of a flexible cable so that the bar appeared either vertical or horizontal. The magnitude of the induced roll vection is taken as the difference between settings of this bar for the stationary and rotating stripes.

The 24 subjects, volunteers from elementary psychology classes, were selected on the basis of suggestibility and screened with a Titmus Vision Tester for a minimum of $20 / 25$ visual acuity. The hypnotic screening was accomplished in a group test in which the subjects listened to a tape recording of a hypnotic induction and then self-rated their responsiveness. Based on these ratings, three groups were chosen. The hypnosis group consisted of eight subjects selected from those who had high scores. The second group, referred to as the simulating group, consisted of eight subjects selected from those with the lowest scores (Orne, 1959). The third group, referred to as the instructed faking group, consisted of four individuals with high scores and four with low scores.

All subjects participated in two further experimental sessions. One was concerned with visual screening, during which eye dominance, near and far visual acuity, and a visual field were determined; in the other session, a visual field was determined and roll vection responses were tested under different instructions of field narrowing. All subjects were tested individually.

The procedure followed in the roll vection session varied with the experimental group. For subjects in the hypnosis group, the nondominant eye was patched and the visual field was measured again, after which the subjects were familiarized with the procedure of adjusting the bar in the roll vection device. Following this, they sat in a reclining chair and were given a cardboard tube to look through in order to demonstrate narrowed vision. This tube limited vision to a field with a diameter of $30.5 \mathrm{deg}$. The tube was then removed, and the subjects listened to a hypnotic induction given by one of the experimenters. At the end of the induction, visual field narrowing was suggested: "Things should appear just as they did when you looked through the tube." After this, a visual field was determined. In all cases, the narrowed visual field was within the stripe-free portion of the field. Then the subjects made a series of six adjustments of the bar in the roll vection device, three each to perceived horizontal and vertical. Of the three settings to either orientation, one was with the surrounding stripes stationary, one with counterclockwise rotation, and one with clockwise rotation. The experimenter read the slant of each adjustment from a calibration disk on the back surface of the perimeter. During the course of the six settings, the subjects were also asked if they could see either the stationary or rotating radial lines in the periphery. They then returned to the reclining chair, were given the suggestion that vision was returning to normal, and were brought out of the trance. A visual field was taken, and the roll vection task was repeated. Finally, they were asked whether they could see the moving or stationary radial lines.

Subjects in the simulating group followed a similar procedure, with the exception that they could not be hypnotized. In accordance with standard practice in experimental hypnosis, the experimenter did not know whether the subjects were hypnotized or simulating. They were informed that the experiment would be terminated if the experimenter suspected that they were not hypnotized. The subjects in the faking group also followed the procedure of the hypnosis group, with the exception that the induction was omitted. Instead, faking subjects were merely asked to perform the experimental tasks as they thought hypnotized subjects with suggestions of field narrowing would. ${ }^{2}$

\section{RESULTS}

Table 1 presents the mean size of the visual field, calculated as the average of two determinations for each of the four principal meridians, before and after narrowing. Because of the low luminance and small 
Table 1

Visual Field Size and Magnitude of Induced Tilt (in Degrees of Arc) Before and After Narrowing Instructions for the Three Experimental Groups

\begin{tabular}{|c|c|c|c|c|c|c|c|c|}
\hline & \multicolumn{4}{|c|}{ Visual Field Size } & \multicolumn{4}{|c|}{ Induced Tilt } \\
\hline & \multicolumn{2}{|c|}{ Before } & \multicolumn{2}{|c|}{ After } & \multicolumn{2}{|c|}{ Before } & \multicolumn{2}{|c|}{ After } \\
\hline & Mean & SD & Mean & SD & Median & Range* & Median & Range* \\
\hline Hypnotized & 32.7 & 13.3 & 15.3 & 13.7 & 8.5 & $3.6-11.9$ & 8.6 & $7.0-12.0$ \\
\hline Simulating & 36.7 & 16.0 & 11.9 & 8.0 & 7.3 & $6.1-11.3$ & 10.1 & $7.8-14.1$ \\
\hline Instructed Faking & 39.3 & 13.4 & 8.7 & 5.0 & 10.3 & $6.0-15.6$ & 10.8 & $7.0-17.5$ \\
\hline
\end{tabular}

*Interquartile range.

angular subtense of the stimulus in the Gambs perimeter, these measures of field size should be considered primarily in terms of their relative values. The field sizes before narrowing are essentially what one would expect from a normal observer under these conditions. It is clear, however, that narrowing instructions dramatically reduced the measures of apparent linear field size. The reductions are $53.2 \%$, $67.6 \%$, and $77.9 \%$ for the hypnosis, simulating, and instructed faking groups, respectively. Thus, in terms of a conventional evaluation of field size, striking reductions are readily obtained by suggestions or special instructions.

In contrast, the data obtained with roll vection, presented as the median value of induced tilt, show no consistent changes of the induced tilt values and are essentially the same before and after narrowing instructions for all three groups. The rather high intersubject variability is typical of roll vection studies.

\section{DISCUSSION}

The results of this study clearly indicate that the failure to report small static stimuli in a perimeter following instructions to narrow the visual fields is independent of the elicitation of roll vection from the same retinal regions. Apparent narrowing, as evaluated by standard perimetry, was easily obtained, but there were no differences in the magnitudes of roll vection following narrowing instructions. These results have implications regarding the use of conventional perimetry in the evaluation of peripheral visual function. In the present instance of visual narrowing, in which subjects denied the presence of visual stimuli in the peripheral fields, the results from the classical perimetry technique would imply a substantial impairment of visual performance. In view of the roll vection results, however, the implication is clear that evaluation of peripheral visual capacity must utilize measures that are relatively insensitive to response bias, demand characteristics, or subjects' expectations.

In the present experiment, the hypnosis, simulating, and faking groups produced similar results with respect to both the degree of apparent visual narrowing and the magnitude of roll vection. The former result suggests the influence of response bias, demand characteristics, or subjects' expectations on verbal responses (and, consequently, apparent visual field size) rather than a true loss of sensory function. Such a view is consistent with the finding of Leibowitz et al. (1980) regarding the relationship between size of hypnotically induced scotomas and viewing distance. The present results are also consistent with those obtained in other hypnotic ablation studies and from observation of hysterics (e.g., Charcot's, 1890 , and Janet's, 1907, patients). Although verbal recognition responses to objects can be eliminated easily, the indirect effects (i.e., those not implicitly related to the task used to define hypnotic visual ablation) of these objects are typically unaffected. Both hypnotized (Orne, 1962) and hysterically "blind" (Jolly, 1892) subjects will walk around obstacles which they cannot "see." Similarly, if the inducing lines of the Ponzo illusion are "ablated," illusion magnitude remains the same (Miller, Hennessy, \& Leibowitz, 1973). It may be that such indirect effects are more appropriate measures of visual loss, in that they are not obviously associated with the task defining the visual loss (i.e., detecting the presence of a target) and are, therefore, less susceptible to response bias, etc. It is evident from the data that measures of the influence of moving visual surrounds on spatial orientation responses are not susceptible to the verbal instructions used in the present study. We are not aware of any studies in which a deliberate attempt was made to alter roll vection responses with verbal instructions. Although this may be possible, it is clearly more difficult than eliminating detection responses to static stimuli, a procedure that we have found to be easily accomplished by suggestion. Other possible indirect measures include circular and linear vection and the influence of visual stimuli on body sway (Dichgans \& Brandt, 1978; Leibowitz \& Dichgans, 1977).

Because of the influence of response bias, demand characteristics, and subjects' expectations on verbal 
reports of peripheral stimuli, it is clear that evaluation of visual impairment needs to be based on more than the criterion of whether a stimulus is reported or not. Leaving aside the problem of denial of peripheral vision, it is also possible that peripheral stimuli of which one is unaware may influerice visual activity. For example, loss of peripheral awareness occurs frequently in everyday life while walking or driving a car. In such cases, attention is directed toward centrally fixated stimuli, while the peripheral visual fields subserve orientation in space without conscious concomitants. This is also illustrated by the ease with which one can walk while reading. Conventional perimetric techniques would accurately detect such loss of awareness, and, as a result, a diagnosis of loss of visual function could be made. However, the loss of visual function reflected in these forms of visual narrowing is unrelate $J$ to some important aspects of visual performance, since many functions of the peripheral visual fieids, in particular spatial orientation, are mediated subconsciously. There is also evidence that visual trauma resulting in loss of the ability to detect the small static targets used in perimetry may not influence the ability to detect movements of large contours (Riddoch, 1917). This ability may be used to mediate orientation in space. To assure a complete assessment of visual function, therefore, perimetry should include measures of spatial orientation ability as well as measures of target detectability. Roll vection may provide a simple means by which spatial orientation ability (which is undetected by conventional perimetry) can be measured.

In summary, traditional perimetric techniques having their basis in verbal reports of the presence or absence of small static targets may be unduly susceptible to response bias, etc., and may result in an overestimation of visual loss. In the present study, roll vection measures were found to be insensitive to instructions not to see peripheral stimuli (which produce the roll vection) and may therefore provide a method relatively free of bias for assessing visual narrowing. In addition, because roll vection provides an index of spatial o,ientation ability, use of this measure in conjunction with conventional perimetry may provide a more complete evaluation of the functional loss accompanying visual field narrowing.

\section{REFERENCE NOTES}

1. Post, R. B., Rodemer, C. S., Dichgans, J., \& Leibowitz, H. W. Dynamic orientation responses are independent of refractive error. Paper presented at the annual meeting of the Association for Research in Vision and Ophthalmology, Sarasota, Florida, 1979.

\section{REFERENCES}

Aulhorn, E., \& Harms, H. Visual perimetry. In D. Jameson \& L. M. Hurvich (Eds.), Handbook of sensory physiology (VII, 4). Heidelberg: Springer, 1972.

Charcot, J. M. Oeuvres complètes. Paris: Bureaux du Progrès Médical, 1890.

Dichgans, J., \& Brandt, T. Visual-vestibular interaction: Effects on self-motion and postural control. In R. Held, H. Leibowitz, \& H. L. Teuber (Eds.), Handbook of sensory physiology (Vol. VIII). Heidelberg: Springer, 1978.

JANET, P. The major symptoms of hysteria. London: Macmillan, 1907

Jolly, F. Über Hysterie bei Kindern. Berliner Klinische Wochenschrift, 1892, 29, 841-845.

Leibowitz, H. Detection of peripheral stimuli under psychological and physiological stress. In Visual search. Washington, D.C: Committee on Vision, National Research CouncilNational Academy of Sciences, 1973.

Leibowitz, H., \& Dichgans, J. Zwei verschiedene Seh-Systeme. Umschau in Wissenschaft und Technik, 1977, 77, 353-354.

Leibowitz, H. W., Lundy, R. M., \& Guez, J. R. The effect of testing distance on suggestion induced visual field narrowing. International Journal of Clinical and Experimental Hypnosis, 1980 , in press.

Miller, R. J., Hennessy, R. T., \& Leibowitz, H. W. The effect of ablation of the background on the magnitude of the Ponzo perspective illusion. International Journal of Clinical and Experimental Hypnosis, 1973, 21, 180-191.

Miller, R. J., \& Leibowitz, H. W. A signal detection analysis of hypnotically induced narrowing of the peripheral visual field. Journal of Abnormal and Social Psychology, 1976, 85, 446-454.

Orne, M. T. The nature of hypnosis: Artifact and essence. Journal of Abnormal and Social Psychology, 1959, 58, 227-299.

OnNe, M. T. Hypnotically induced hallucinations. In L. J. West (Ed.), Hallucinations. New York: Grune and Stratton, 1962.

Post, R. B., \& Leibowitz, H. W. The independence of radial localization from refractive error. Journal of the Optical Society of America, in press.

$\mathbf{R}_{1 \text { DDoch, }}$ G. Dissociation of visual perception due to occipital injuries with special reference to appreciation of movement. Brain, 1917, 40, 15-57.

\section{NOTES}

1. This phenomenon is often referred to as "tunnel" vision. However, in the medical literature, this term is often associated with visual loss resulting from retinal pathology.

2. As a control for the possible effect of time upon the measures being investigated, another group first listened to an experimenter reading stories aloud for $15 \mathrm{~min}$ prior to the first field and roll vection tests. Following this, they listened for $5 \mathrm{~min}$ more to the experimenter reading aloud and then were rutested on the vection device and perimeter. This group was informed only after the test that they were participating in a study of hypnosis and visual narrowing. The means and intersubject variability were highly similar to those of the other three groups.

(Received for publication June 21, 1979; revision accepted March 28, 1980.) 